\title{
Numerical simulation of microbial depolymerization process of exogenous type
}

\author{
M. Watanabe* F. Kawai ${ }^{\dagger}$
}

(Received 31 October 2004, revised 20 October 2005)

\begin{abstract}
A microbial depolymerization process of exogenous type is studied with experimental data introduced into the analysis. A mathematical model for exogenous depolymerization processes is described together with the numerical techniques to analyze it. Given an initial weight distribution of polyethylene glycol with respect to the molecular weight and its final weight distribution obtained after cultivation of a microbial consortium E1, an inverse problem of an initial value problem determines an oxidation rate. Given the oxidation rate, the initial value problem was solved to simulate the transition of weight distribution. A numerical simulation shows that the mathematical method
\end{abstract}

${ }^{*}$ Faculty of Environmental Science and Technology, Okayama University, 1-1, Naka 3-chome, Tsushima, Okayama 700-8530, JAPAN.

mailto: watanabe@ems.okayama-u.ac.jp

${ }^{\dagger}$ Research Institute for Bioresources, Okayama University

See http://anziamj.austms.org.au/V46/CTAC2004/Wata for this article, (C) Austral. Mathematical Soc. 2005. Published November 8, 2005. ISSN 1446-8735 
of analysis of an exogenous depolymerization process is practically acceptable. The numerical techniques illustrated here are applicable to the analyses of other exogenous depolymerization processes.

\section{Contents}

1 Introduction

C1189

2 Exogenous depolymerization model

C1192

3 Computational analysis of inverse problem

C1194

4 Simulation of PEG biodegradation

C1197

5 Discussion

C1198

References

C1201

\section{Introduction}

In general, microbial depolymerization processes fall into one of two categories: exogenous type or endogenous type. In an exogenous depolymerization process, polymers are depolymerized by separation of monomer units at their terminals. Examples include biodegradation processes of polyethylene (PE). $\mathrm{PE}$ is structurally a long-chain alkane (basically normal-type). The initial step of the oxidation of $n$-alkanes is hydroxylation to provide the corresponding primary (or secondary) alcohol, which is oxidized further to an aldehyde (or ketone) and then to an acid. Carboxylated $n$-alkanes are structurally analogous to fatty acids, and should be subject to $\beta$-oxidation processes to produce depolymerized alkanoic acids by two-carbon units (acetic acid). A mathematical model has been proposed and analyzed to study the 
PE biodegradation which involves the $\beta$-oxidation. $[1,2,9,7,8]$. Unlike exogenous depolymerization processes, where oxidation takes a substantial part, hydrolysis is often involved in a depolymerization process of endogenous type, in which internal cleavages take place. The enzymatic degradation of polyvinyl alcohol (PVA) is an example of endogenous depolymerization. PVA is a carbon-chain polymer with a hydroxyl group attached to every other carbon unit, which are known to be degraded by random oxidation of hydroxyl groups and hydrolysis of mono/diketones [3, 12, 13]. A mathematical model was also proposed and analyzed to study the endogenous depolymerization of PVA $[6,7,10]$.

Here, the analytical and numerical study of exogenous depolymerization is continued to cover the biodegradation of polyethylene glycol (PEG). PEG is one of polyethers that are utilized for constituents in a number of products including lubricants, antifreeze agents, inks, and cosmetics, and also for raw materials to synthesize detergents or polyurethanes. Metabolism of polyethers has been well documented with regards to PEG. It is depolymerized exogenously by liberating $\mathrm{C}_{2}$ compounds, either aerobically or anaerobically $[3,4,12]$. The microbial consortium E-1 was cultivated on a culture medium containing PEG as its sole carbon source. High performance liquid chromatography (HPLC) patterns were analyzed to obtain the initial weight distribution of PEG with respect to the molecular weight as well as the weight distribution after cultivation for one day and three days; they are shown in Figure 1. These experimental results were introduced into the mathematical analysis of PEG biodegradation.

In the following sections, the biodegradability of PEG is examined by analyzing its weight distribution obtained before and after cultivation of a microbial consortium E-1. An inverse problem of an initial value problem was solved numerically to determine an oxidation rate for which the solution of the initial value problem also satisfies a final condition. A result of a numerical simulation is presented to show that the mathematical model is appropriate for the PEG biodegradation. The significance of the outcome 


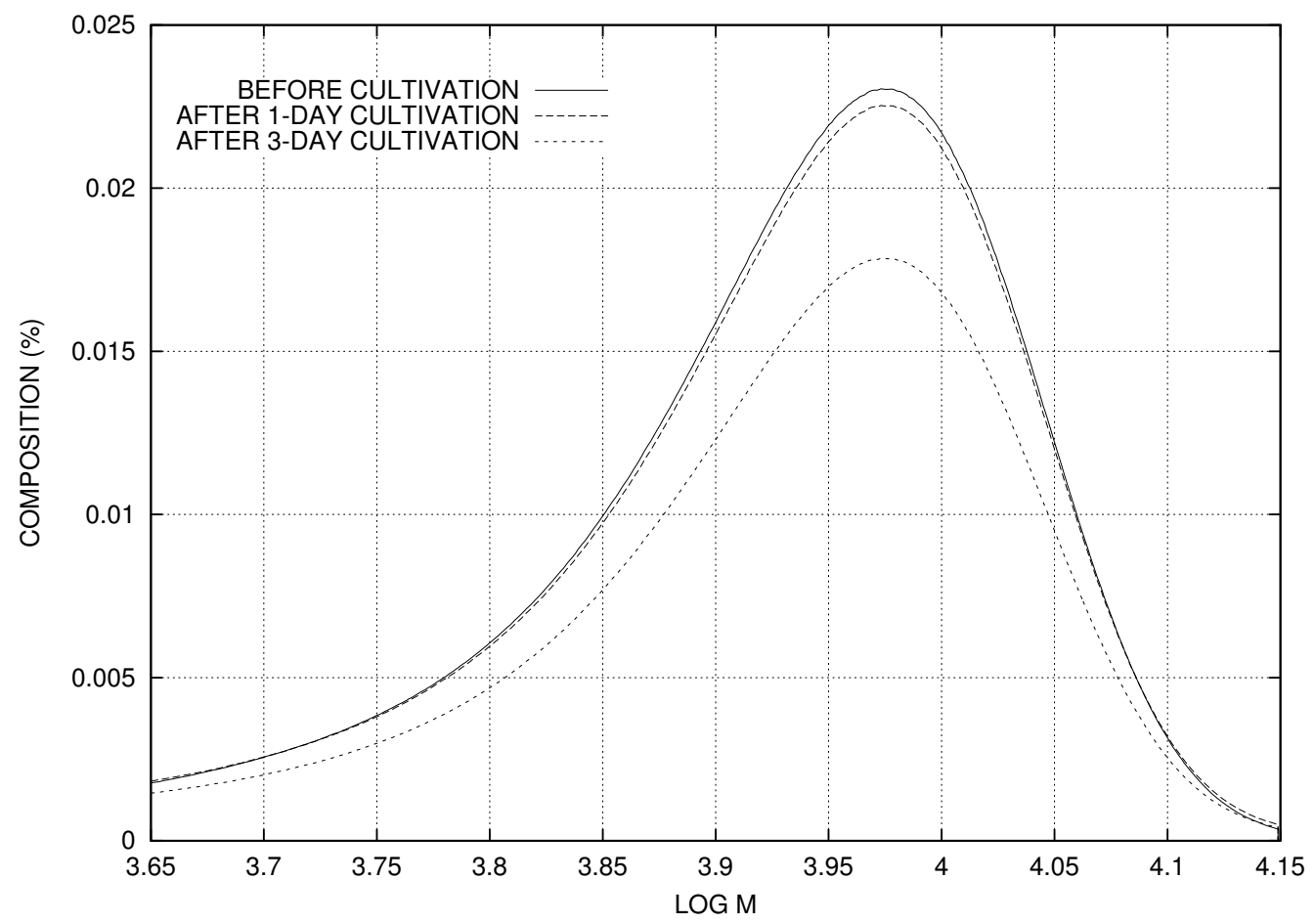

FIgURE 1: The weight distribution of PEG before and after 1 day and 3 day cultivation of the microbial consortium E-1. 
is discussed, and the dependence of PEG biodegradability on the molecular weight examined.

\section{Exogenous depolymerization model}

The PEG biodegradation is presumed to follow the exogenous depolymerization model, which is originally developed for the PE biodegradation. It is based on the assumption that a molecule loses its weight by a fixed amount per unit time, that some small molecules are directly consumed by microorganisms, and that the consumption rate depends on the sizes of molecules. Those assumptions lead to the following mathematical model of PE biodegradation $[1,2,8]$.

$$
\frac{d x}{d t}=-\alpha(M) x+\beta(M+L) \frac{M}{M+L} y,
$$

where $\alpha(M)=\rho(M)+\beta(M)$. Here $t$ and $M$ represent the cultivation time and the molecular weight respectively, and $x=w(t, M)$ denotes the total weight of $M$-molecules (the PE molecules with molecular weight $M$ ) present at time $t$. The parameter $L$ represents the amount of the weight loss due to the terminal separation. The variable $y=w(t, M+L)$, the total weight of $(M+L)$-molecules present at time $t$. The function $\rho(M)$ represents the direct consumption rate per unit time, and the function $\beta(M)$ represents the weight conversion rate per unit time from the class of $M$-molecules to the class of $(M-L)$-molecules. This model is motivated by two essential factors in PE biodegradation: the gradual weight loss of large molecules due to the terminal separation ( $\beta$-oxidation); and the direct consumption of small molecules by cells. The left-hand side of the equation (1) represents the time rate of change in the total weight of $M$-molecules. The first term on the right-hand side of the equation (1) represents the amount lost due to the direct consumption and the terminal separation in the total weight of $M$ molecules per unit time, and the second term represents the amount gained due to the terminal separation of $M+L$-molecules per unit time. 
The mathematical model (1), which was originally developed for the PE biodegradation, can be viewed as a general biodegradation model involving exogenous depolymerization processes. A PEG molecule is first oxidized at its terminal, and an ether bond is split. This process corresponds to $\beta$-oxidation in the PE biodegradation. We call this process oxidation, because oxidation is involved throughout its depolymerization process [4]. Note that $L=44$ $\left(\mathrm{CH}_{2} \mathrm{CH}_{2} \mathrm{O}\right)$ in the exogenous depolymerization of PEG.

The solution $x=w(t, M)$ of (1) is associated with the initial condition

$$
w(0, M)=f(M) .
$$

Here, $f(M)$ is some prescribed function that represents the initial weight distribution. Given the total consumption rate and the oxidation rate, the equation (1) and the initial condition (2) form an initial value problem to find the unknown function $w(t, M)$. On the other hand, given the initial condition (2) and an additional final condition at $t=T>0$,

$$
w(T, M)=g(M),
$$

an inverse problem determines the degradation rates $\alpha(M)$ and $\beta(M)$ for which the solution $w(t, M)$ of the initial value problem (1) and (2) also satisfies the final condition (3).

The condition

$$
0<g(M)<f(M)+\frac{M \beta(M+L)}{M+L} \int_{0}^{T} w(s, M+L) d s .
$$

is a sufficient condition for a unique positive total degradation rate $\alpha(M)$ to exist, given the $\beta$-oxidation rate $\beta(M+L)$ and the weight distribution $w(t, M+L)[8]$ :

Note that molecules must penetrate through the membranes to enter into the cells in order to become subject to the direct consumption. On the other hand, the rate of the membrane transport decreases as the molecular size 
increases. Moreover, there should be a value of the molecular weight, which we call $M_{\rho}$, such that $\rho(M)=0$ for $M \geq M_{\rho}$. Since $\alpha(M)=\rho(M)+\beta(M)$,

$$
\alpha(M)=\beta(M)
$$

for $M \geq M_{\rho}$. We deal with the case where the molecular weight is so large that the equation (5) holds. Here the value of $M_{\rho}$ is assumed to lie in range from 1000 to 2000 , and the analysis is focused on the molecular weight over $10^{3.63}$.

\section{Computational analysis of inverse problem}

Here a numerical scheme developed by Watanabe et al. [9] to solve the inverse problem is introduced, and a numerical result presented. In order to solve the inverse problem, cover the domain $[a, b]$ with the intervals of length $L:[a+i L, a+(i+1) L], i=0, \ldots, l-1$, extending the definition of $f(M)$ and $g(M)$ if necessary. Here the positive integer $l$ satisfies the condition

$$
\frac{b-a}{L} \leq l<\frac{b-a}{L}+1 \text {. }
$$

Choose a positive integer $m$, and set (with $\delta M=L / m$ )

$$
M_{i, j}=a+i L+j \delta M, \quad i=0,1, \ldots, l-1, \quad j=0,1, \ldots, m .
$$

Denote by $\alpha_{i, j}$ and $\beta_{i, j}$ approximate values of $\alpha\left(M_{i, j}\right)$ and $\beta\left(M_{i, j}\right)$, respectively. Note that $\alpha_{i, j}=\beta_{i, j}, 0 \leq i \leq l, 0 \leq j \leq m$, in view of equation (5). Let $f_{i, j}=f\left(M_{i, j}\right), g_{i, j}=g\left(M_{i, j}\right)$. Choose a positive integer $n$, and set

$$
t_{k}=k \delta t, \quad k=0,1,2, \ldots, n \quad\left(\delta t=\frac{T}{n}\right)
$$

so that the time interval $[0, T]$ is divided into $n$ intervals: $\left[t_{k-1}, t_{k}\right], k=$ $1,2, \ldots, n$. Let $w_{i, j, k}$ denote an approximate value of $w\left(t_{k}, M_{i, j}\right)$. Given $w_{i+1, j, k}$, 
$j=m-1, m-2, \ldots, 0, k=0,1, \ldots, n$, and $\beta_{i+1, j}, j=m-1, m-2, \ldots, 0$, $\eta=\alpha_{i, j}$ is a solution of the equation

$$
\Phi_{i, j}(\eta)=0
$$

where

$\Phi_{i, j}(\eta)=e^{-\eta T}\left[f_{i, j}+\frac{\delta t \beta_{i+1, j} M_{i, j}}{2 M_{i+1, j}} \sum_{k=1}^{n}\left(e^{\eta t_{k-1}} w_{i+1, j, k-1}+e^{\eta t_{k}} w_{i+1, j, k}\right)\right]-g_{i, j}$

Start the numerical process at $i=l-1$ setting appropriate values of $w_{l, j, k}$, $j=0,1, \ldots, m-1, k=0,1, \ldots, n$, and march backward: $i=l-1, l-$ $2, \ldots, 0$. At each step, evaluate a numerical solution $\eta=\alpha_{i, j}$ of (6) by Newton's method for $j=m-1, m-2, \ldots, 0$, examining the condition

$$
0<g_{i, j}<f_{i, j}+\frac{\delta t \beta_{i+1, j} M_{i, j}}{2 M_{i+1, j}} \sum_{k=1}^{n}\left(w_{i+1, j, k-1}+w_{i+1, j, k}\right),
$$

which is a sufficient condition for the equation (6) to have a unique positive solution $\eta=\alpha_{i, j}$. Once an approximate solution $\eta=\alpha_{i, j}$ of equation (6) is found, approximate values $w_{i, j, k}$ of $w\left(t_{k}, M_{i, j}\right)$ are

$$
w_{i, j, k}=e^{-\eta t_{k}} f_{i, j}+\frac{e^{-\eta t_{k}} \delta t \beta_{i+1, j} M_{i, j}}{2 M_{i+1, j}} \sum_{p=1}^{k}\left(e^{\eta t_{p-1}} w_{i+1, j, p-1}+e^{\eta t_{p}} w_{i+1, j, p}\right)
$$

with $\eta=\alpha_{i, j}$ for $k=0,1, \ldots, n$.

The inverse problem was solved numerically to find the exogenous degradation rate $\beta(M)$ based on the initial weight distribution and the final weight distribution shown in Figure 2 with the values of the parameters: $L=44$, $T=3, n=1200, m=10$. The values of the parameters $a=10^{3.63} \approx 4266$, $b=10^{4.15} \approx 14125$, and $l=225$ were also set. Figure 2 shows the graph of the exogenous degradation rate generated numerically. 


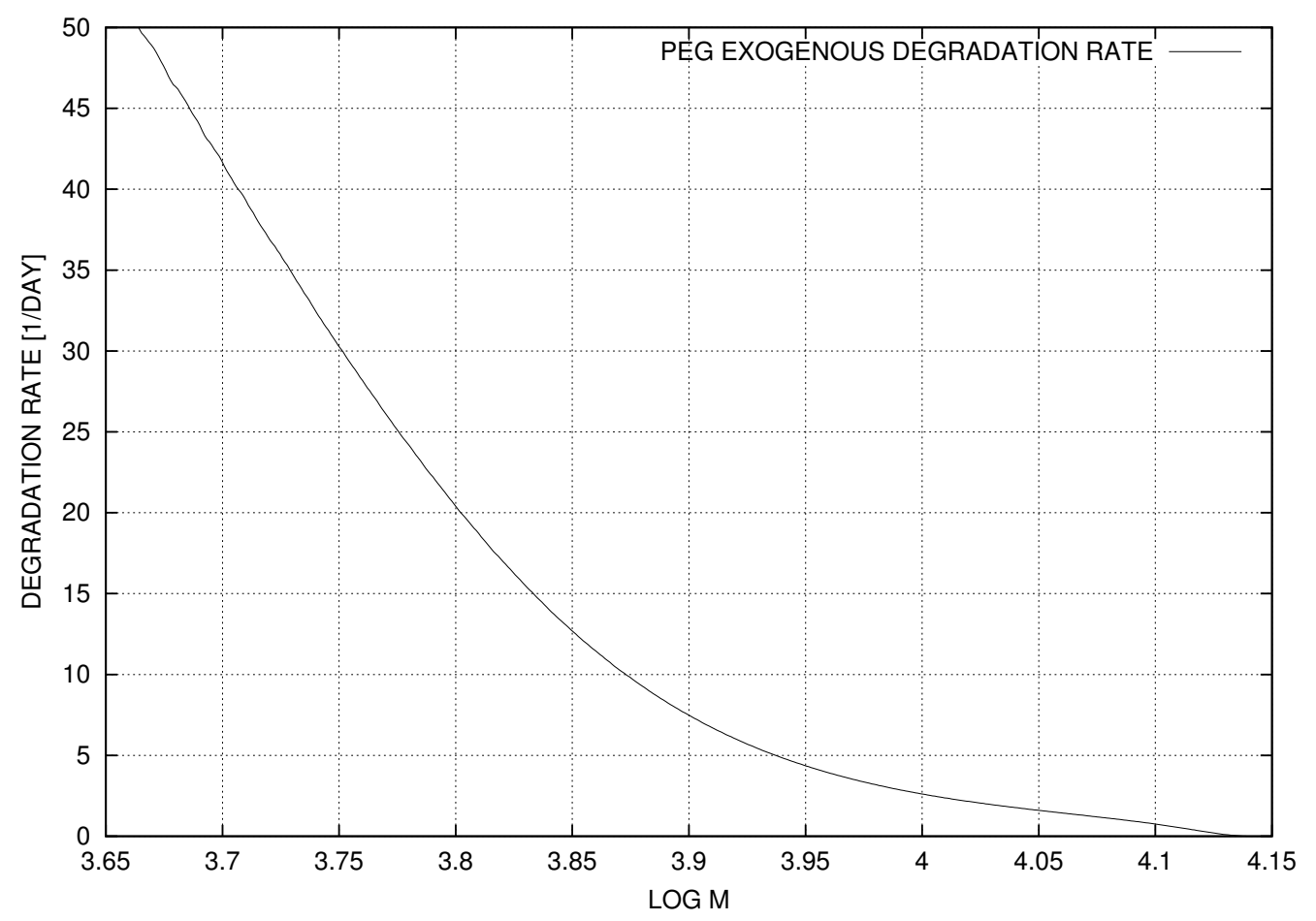

FiguRE 2: The exogenous degradation rate $\beta(M)$ based on HPLC profiles obtained before and after 3 day cultivation of the microbial consortium E-1 (Figure 1). 


\section{Simulation of PEG biodegradation}

In the process of evaluating the exogenous degradation rate, the approximate values $w_{i, j, k}$ of $w(t, M)$ at $t=t_{k}$ and $M=M_{i, j}$ are also evaluated. On the other hand, once the approximate values of the total consumption rates and the $\beta$-oxidation rates are given, the initial value problem (1) and (2) can be solved directly to see how well the numerical results and the experimental results agree. Here the initial value problem was solved numerically with techniques developed previously [2, 8, 9].

Choose a positive integer $N$ and set

$$
M_{i}=a+i \Delta M, \quad i=0,1,2, \ldots, N \quad\left(\Delta M=\frac{b-a}{N}\right) .
$$

An approximate solution of the differential equation (1) at $M=M_{i}$ is denoted by $w_{i}=w_{i}(t), i=0,1,2, \ldots, N$. There is a non-negative integer $K$ and a constant $R$ such that $L=K \Delta M+R, 0 \leq R<\Delta M$, and that the inequalities

$$
M_{i+K} \leq M_{i}+L<M_{i+K+1}
$$

hold. Then approximate values of $w\left(t, M_{i}+L\right)$ and $\beta\left(M_{i}+L\right)$ are obtained using

$$
\begin{aligned}
& w\left(t, M_{i}+L\right) \approx\left(1-\frac{R}{\Delta M}\right) w\left(t, M_{i+K}\right)+\frac{R}{\Delta M} w\left(t, M_{i+K+1}\right), \\
& \beta\left(M_{i}+L\right) \approx\left(1-\frac{R}{\Delta M}\right) \beta\left(M_{i+K}\right)+\frac{R}{\Delta M} \beta\left(M_{i+K+1}\right) .
\end{aligned}
$$

Substituting these expressions in the differential equation (1) and setting $M=M_{i}$, we obtain the linear system:

$$
\frac{d w_{i}}{d t}=-\alpha_{i} w_{i}+\beta_{i} w_{i+K}+\gamma_{i} w_{i+K+1}, \quad i=0,1,2, \ldots, N .
$$


Here the coefficients

$$
\begin{aligned}
& \alpha_{i}=\alpha\left(M_{i}\right), \quad \beta_{i}=\sigma_{i} \frac{M_{i}}{M_{i}+L}\left(1-\frac{R}{\Delta M}\right), \quad \gamma_{i}=\sigma_{i} \frac{M_{i}}{M_{i}+L} \cdot \frac{R}{\Delta M}, \\
& \text { and } \quad \sigma_{i}=\left(1-\frac{R}{\Delta M}\right) \beta\left(M_{i+K}\right)+\frac{R}{\Delta M} \beta\left(M_{i+K+1}\right) .
\end{aligned}
$$

Approximate values of the degradation rates $\alpha\left(M_{i}\right)$ and $\beta\left(M_{i}\right)$ are linear approximations in terms of the approximate values $\alpha_{j, k}$ and $\beta_{j, k}$ obtained by solving the inverse problem.

For all sufficiently large $M$, the oxidation rate becomes 0 . In particular, we assume that the last two terms on the right-hand side of the equation (7) are absent when $i+K$ exceeds $N$, so that the system (7) becomes a closed system to be solved for unknown functions $w_{i}=w_{i}(t), i=0,1,2, \ldots, N$. In view of the condition (2), these functions are subject to the initial condition

$$
w_{i}(0)=f_{i}=f\left(M_{i}\right) .
$$

Given the initial weight distribution shown in Figure 1, and the corresponding degradation rate shown in Figure 2, the initial value problem (7) and (8) was solved numerically implementing the fourth-order Adams-BashforthMoulton predictor-corrector in PECE mode in conjunction with the classical fourth order Runge-Kutta method to generate approximate solutions in the first three steps [5] by using $N=10000$, and a time interval $\Delta t=0.0025$. Figure 3 shows the simulated transition of the weight distribution during cultivation of the microbial consortium E-1. Note that the experimental result obtained after cultivation for three days and the corresponding numerical result are almost indistinguishable.

\section{Discussion}

Recall that the oxidation rate $\beta(M)$ is the ratio of the total weight of $M$ molecules degraded to the total weight of entire $M$-molecules per unit time. 


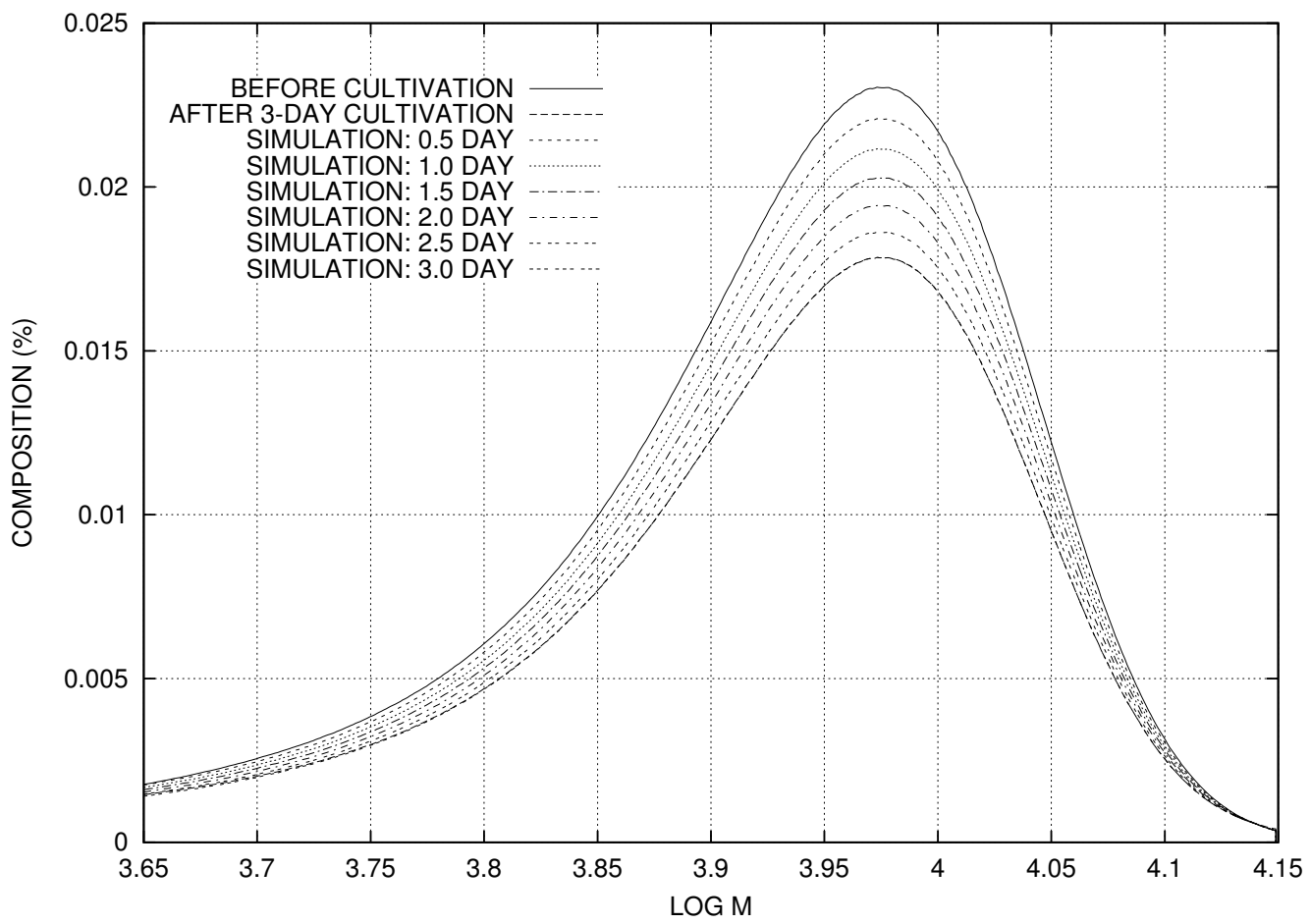

Figure 3: Transition of the Weight distribution of PEG during 3-day cultivation of the microbial consortium E-1. 
It also represents the ratio of the number of $M$-molecules that undergo exogenous depolymerization processes. Since the reciprocal of $\beta(M)$ can be regarded as the average time it takes for one cycle of exogenous depolymerization process to be completed, the oxidation rate $\beta(M)$ might be presumed independent of the molecular size $M$, for the exogenous depolymerization processes take place only at the terminals of the molecules. However, in practice this is the case only for molecules of moderate sizes.

As the metabolic enzymes are located in cell membranes, the exogenous depolymerization processes take place inside a cell, and at least one end of a molecule must penetrate through its outer membrane to become subject to an exogenous depolymerization process in the periplasm. When a molecule makes contact with a microorganism, a part of a fixed length should be taken into a cell in the fixed period. If that part contains one of the terminals, the enzyme takes effect and the exogenous biodegradation process starts. The possibility for a part of a fixed length to contain a terminal becomes less when the molecule becomes large. The result shown in Figure 2 clearly indicates the dependence of the rate of membrane transport with respect to the molecular weight, and our mathematical analysis reveals the role of membrane transport in exogenous depolymerization processes.

The exogenous depolymerization model originally developed for PE biodegradation has been applied successfully to PEG biodegradation. The inverse problem has been solved numerically to find the oxidation rate. The validity of the numerical result has been confirmed by the numerical simulation. Different HPLC patterns obtained before and after cultivation of a microbial consortium E-1 were taken into mathematical analysis of PEG biodegradation [11]. However, the weight distributions shown in Figure 3 were based on HPLC patterns recently obtained, and this is the first time they are presented.

What is shown in Figure 2 should be viewed as the average degradation rate over three days of cultivation. It is valid only for the degradation rate close to three days. Figure 4 shows the difference between the actual mea- 
surement and the numerical result based on the degradation rate shown in Figure 2 for the weight distribution after one day of cultivation. Note that the numerical result overshoots the experimental result. The degradation rate depends on the microbial population, and it was tacitly assumed to be constant by setting the degradation rate independent of time. It can be assumed to be constant after the microbial population reaches the steady state, and Figure 4 shows that it takes more than a few days to reach that stage. In the study of PEwax biodegradation, the weight distribution of PEwax after five weeks cultivation was simulated using the time-independent degradation rate based on the weight distribution before and after three week cultivation of the fungus Aspergillus sp. AK-3 [9], and the difference between the experimental result and the numerical result was acceptable. That shows that the microbial population reached the steady state after three weeks of cultivation, and the assumption that the degradation rate was independent of time was in fact valid for the weight distribution after three weeks of cultivation. What is shown in Figure 4 is that the microbial population is still increasing in the first three days of cultivation, and that the degradation rate should be time-dependent at least in that period.

Acknowledgments: The authors thank Ms. Y. Shimizu (Research Institute for Bioresources, Okayama University) for her technical support. This work was supported in part by a JSPS (MEXT) Grant-in-Aid for Scientific Research (C) (16540106).

\section{References}

[1] Fusako Kawai, Masaji Watanabe, Masaru Shibata, Shigeo Yokoyama, Yasuhiro Sudate, Experimental analysis and numerical simulation for biodegradability of polyethylene, Polymer Degradation and Stability 76 (2002) 129-135. C1190, C1192 


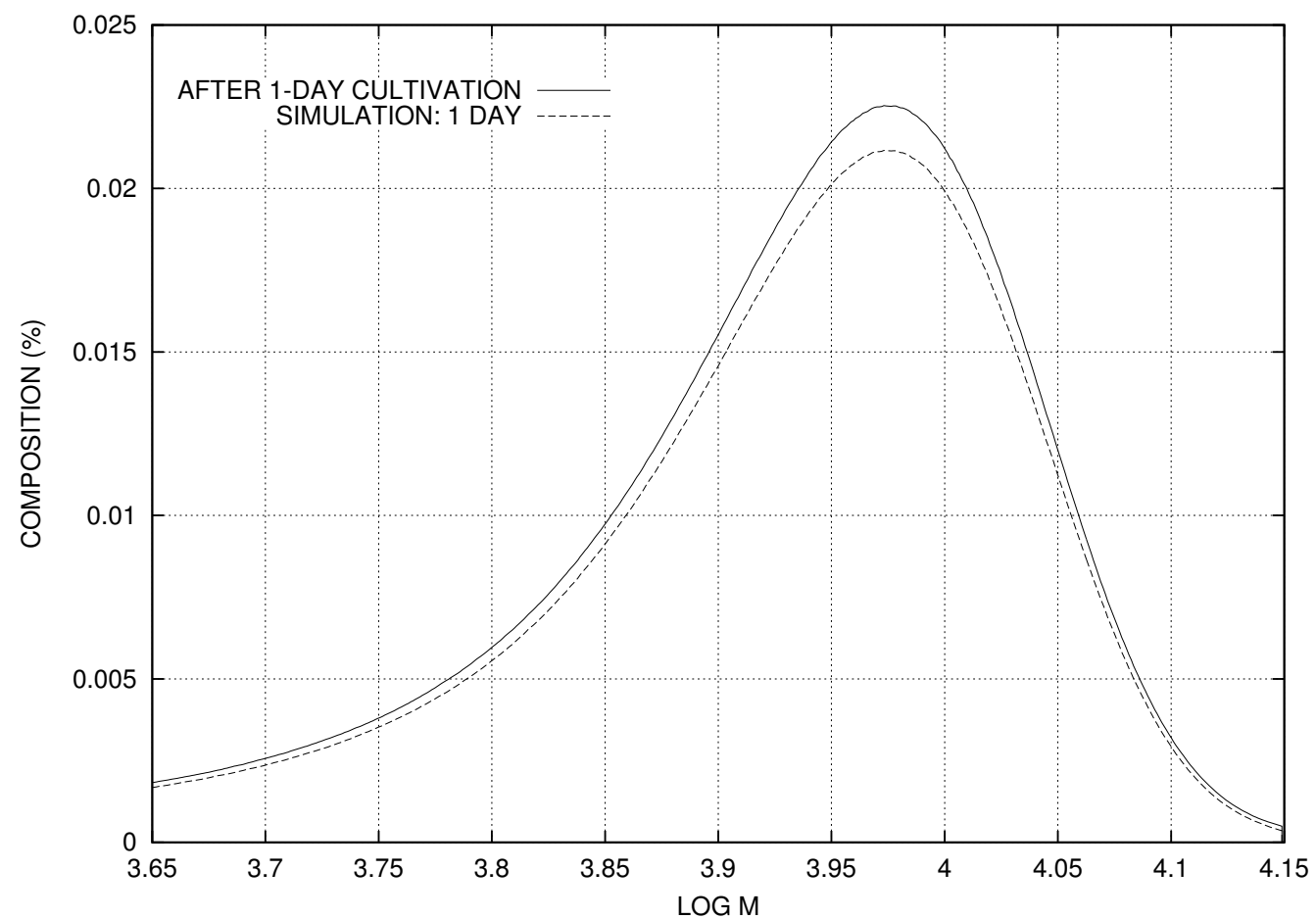

FiguRe 4: Experimental result and numerical result based on the degradation rate shown in Figure 2 for Weight distribution of PEG after 1-day cultivation of the microbial consortium E-1. 
[2] Masaji Watanabe, Fusako Kawai, Masaru Shibata, Shigeo Yokoyama, Yasuhiro Sudate, Computational method for analysis of polyethylene biodegradation, Journal of Computational and Applied Mathematics, Volume 161, Issue 1, 1 December 2003, 133-144.

http://dx.doi.org/10.1016/S0377-0427(03)00551-X C1190, C1192, C1197

[3] Fusako Kawai, Breakdown of plastics and polymers by microorganisms, Advances in Biochemical Engineering/Biotechnology, Vol. 52, 151-194 (1995). C1190

[4] F. Kawai, Microbial degradation of polyethers, Applied Microbiology and Biotechnology (2002) 58:30-38. http://dx.doi.org/10.1007/s00253-001-0850-2 C1190, C1193

[5] J. D. Lambert, Computational Methods in Ordinary Differential Equations, John Wiley Sons, Chichester, 1973. C1198

[6] Masaji Watanabe, Fusako Kawai, Numerical Simulation for Enzymatic Degradation of Poly(vinyl Alcohol), Polymer Degradation and Stability, Volume 81, Issue 3, 2003, 393-399. http://dx.doi.org/10.1016/S0141-3910(03)00122-8 C1190

[7] Masaji Watanabe and Fusako Kawai, Analysis of polymeric biodegradability based on experimental results and numerical simulation, Environmental Research and Control (2003), 25, 25-32 (In Japanese). C1190

[8] Fusako Kawai, Masaji Watanabe, Masaru Shibata, Shigeo Yokoyama, Yasuhiro Sudate, Shizue Hayashi, Comparative study on biodegradability of polyethylene wax by bacteria and fungi, Polymer Degradation and Stability 86 (2004), 105-114. http://dx.doi.org/10.1016/j.polymdegradstab.2004.03.015 C1190, C1192, C1193, C1197 
[9] Masaji Watanabe, Fusako Kawai, Masaru Shibata, Shigeo Yokoyama, Yasuhiro Sudate, Shizue Hayashi, Analytical and computational techniques for exogenous depolymerization of xenobiotic polymers, Mathematical Biosciences 192 (2004) 19-37. http://dx.doi.org/10.1016/j.mbs.2004.06.006 C1190, C1194, C1197, C1201

[10] Masaji Watanabe, Fusako Kawai, Mathematical modelling and computational analysis of enzymatic degradation of xenobiotic polymers, submitted for publication. C1190

[11] Masaji Watanabe, Fusako Kawai, Analysis of biodegradability for polyethylene glycol via numerical simulation, Environmental Research and Control 26 (2004), 17-22 (in Japanese). C1200

[12] F. Kawai, Xenobiotic polymers, in: T. Imanaka, ed., Great Development of Microorganisms, (NTS. Inc., Tokyo, 2002) 865-870 (in Japanese). C1190

[13] S. Matsumura, N. Tomizawa, A. Toki, K. Nishikawa and K. Toshima, Novel Poly(vinyl alcohol)-degrading enzyme and the degradation mechanism, Macromolecules 32 (1999), 7753-7761. C1190 